Running head: LONGITUDINAL COVID FEAR

\title{
Pandemic panic? Results of a 14-month longitudinal study on fear of COVID-19
}

This preprint has now been published as: Mertens, G., Lodder, P., Smeets, T., \& Duijndam, S. (2022). Pandemic panic? Results of a 14-month longitudinal study on fear of COVID-19. Journal of Affective Disorders, 322, 15-23. https://doi.org/10.1016/j.jad.2022.11.008

Please refer to this article for the final version of the manuscript

Gaëtan Mertens ${ }^{1}$, Paul Lodder ${ }^{1,2}$, Tom Smeets ${ }^{1}, \&$ Stefanie Duijndam ${ }^{1}$

${ }^{1}$ Department of Medical and Clinical Psychology, Tilburg University, Tilburg, the Netherlands

${ }^{2}$ Department of Methodology and Statistics, Tilburg University, Tilburg, the Netherlands

Word count main text (excluding title page, abstract, tables, and references): 4859

Correspondence concerning this article should be addressed to Gaëtan Mertens, Department of Medical and Clinical Psychology, Warandelaan 2, room T526, Tilburg University, 5037AB Tilburg, the Netherlands.

E-mail: g.mertens@tilburguniversity.edu

Tel: +31134663757 
Declarations of Conflicting Interests: The authors declared no conflicts of interest.

Acknowledgements: This research was funded by a fast-track coronavirus grant (440.20.037) awarded to Gaëtan Mertens by the Dutch Research Council. 


\begin{abstract}
Background: Fear is an evolutionary adaptive emotion that serves to protect the organism from harm. Once a threat diminishes, fear should also dissipate as otherwise fear may become chronic and pathological. While actual threat of the COVID-19 pandemic (i.e., number of infections, hospitalizations, and deaths) has substantially varied over the course of the pandemic, it remains unclear whether (subjective) fear has followed a similar pattern.
\end{abstract}

Method: To examine the development of fear of COVID-19 during the pandemic and investigate potential predictors of chronic fear, we conducted a large online longitudinal study ( $N$ = 2000) using the Prolific platform between April 2020 and June 2021. Participants were voluntary response samples and consisted of residents of 34 different countries. The Fear of the Coronavirus Questionnaire (FCQ) and several other demographic and psychological measures were completed monthly.

Results: Overall, we find that fear steadily decreased since April 2020. Additional analyses showed that elevated fear was predicted by region (i.e., North America > Europe), anxious traits, gender, risks for loved ones, general health, and media use.

Limitations: The interpretation of the results of this study is limited by the nonrepresentativeness of the sample and the lack of data points between August 2020 and June 2021. Conclusions: This study helps to characterize the trajectory of fear levels throughout the COVID-19 pandemic and establish several relevant predictors of increased fear.

Keywords: Fear; COVID-19; Media; Intolerance of uncertainty; Health anxiety 


\section{Introduction}

The SARS-CoV-2 virus causing the coronavirus disease 2019 (COVID-19) was first identified in December 2019 in Wuhan, China. Since that time, it has spread pandemically, resulting in over 600 million confirmed cases and more than 6.5 million deaths worldwide at the time of writing (September 2022) (Dong et al., 2020). In addition, millions of people are affected by social restrictions imposed by governments, such as social distancing or lockdowns, severely interfering with daily life and employment. Unsurprisingly, many researchers have linked the COVID-19 pandemic to mental health issues such as anxiety-related disorders, depression, and post-traumatic stress (Lu et al., 2020; Sciensano, 2020; Taylor et al., 2020).

Fear is a central emotion for explaining the mental health consequences of the COVID-19 pandemic (Mertens et al., 2020). Fear is an adaptive emotion that serves to protect the organism from potential danger. However, when fear is excessive or chronic, it can lead to substantial suffering. Several studies have found specific worries and fear relating to the COVID-19 pandemic (Ahorsu et al., 2020; Mertens et al., 2020) and these have been linked to increases in anxiety, stress and depression (Fitzpatrick et al., 2020; Lee et al., 2020; Montano \& Acebes, 2020). Nonetheless, COVID-19 related fear does not only have negative consequences. In fact, increased fear is related to increased preventive behaviors in relation to the coronavirus, such as hand washing and social distancing (Harper et al., 2020), which help in reducing the spread of the virus. Given these negative and positive effects of COVID-19 related fear, the present study investigates the development of fear throughout the first one-and-a-half year of the pandemic and examined potential predictors of increased fear.

Several factors may contribute to heightened fear of COVID-19. First, there may be differences in the objective risks of infection by the coronavirus. So far, some countries and 
regions have been more heavily affected by the coronavirus than others (e.g., see https://ourworldindata.org/coronavirus). For instance, several media reports have noted a substantial difference in the number of cases between the United States (US) and the European Union (EU) in the summer months of 2020 (Nisen, 2020; Winkleman \& Santamaria, 2020). Particularly, while the number of daily new infections in the EU steadily decreased for several months after the first wave of the pandemic in March-April 2020, the number of infections remained high during this period in the US. This may translate into different levels of threat perception and fear in these two regions.

Another predictor of increased fear may be inter-individual differences in anxiousness. Some individuals may be more prone than others to experience heightened fear for COVID-19. A relevant psychological factor may be health anxiety, which refers to the heightened tendency of individuals to interpret benign bodily sensations as if one has or is contracting a serious illness (Salkovskis et al., 2002). Given that some COVID-19 symptoms are similar to those of a common cold or regular flu, it is likely that individuals high in health anxiety will more rapidly experience heightened fear about having contracted the virus and of suffering serious (long-term) health consequences (Asmundson \& Taylor, 2020). In addition, given that there are many unknowns relating to the coronavirus, such as how this new virus will spread and what the socioeconomic consequences of this pandemic are, uncertainty may also play an important role in fear of COVID-19. Particularly, individual differences in the tendency to find uncertain situations and a lack of information intolerable (i.e., intolerance of uncertainty, which is related to different types of anxiety disorders such as generalized anxiety disorder and panic disorder; see Carleton, 2016) may be positively related to fear of COVID-19. It is worthwhile to note that there are many other related constructs that may be of relevance to predict levels of fear of COVID-19 
(e.g., neuroticism, trait anxiety, insecure attachment, etc.) (De Landsheer \& Walburg, 2022; Troisi et al., 2021). However, we decided to focus on health anxiety and intolerance of uncertainty in the current study given their relevance for the perceived threat of infection and physical symptoms related to COVID-19 and the broad uncertainty caused by the pandemic, respectively.

Finally, usage of media may be related to heightened fear of COVID-19. Such a link between increased media usage and heightened fear has been found in previous pandemic outbreaks (Van den Bulck \& Custers, 2009) and also for the current COVID-19 pandemic (Chao et al., 2020; Hamidein et al., 2020; Jungmann \& Witthöft, 2020). This may be because news coverage of the COVID-19 pandemic tended to be particularly negative in sentiment (52\% of the headlines) and heavily focused on fear (20\% of the headlines) (Aslam et al., 2020).

Taken together, the aim of this study was to investigate the development of COVID-19 specific fear throughout the first six months of the COVID-19 pandemic, and with an additional follow-up one year later. Additionally, we wanted to establish possible predictors of elevated and/or chronic fear during this period. Therefore, we conducted a large longitudinal online survey $(N=2000)$ between April 2020 and June 2021 in which a voluntary response sample completed the Fear of the Coronavirus Questionnaires (FCQ; Mertens, Gerritsen, et al., 2020) together with several relevant predictors for increased fear of COVID-19 (i.e., country of residence, health anxiety, intolerance of uncertainty, media use, and demographic variables). 


\section{Methods}

\subsection{Participants and sample size determination}

For this study, the data of two samples were used. The first sample was a predominantly Dutch sample of 439 participants who completed the survey between $13^{\text {th }}$ and $17^{\text {th }}$ of March 2020 . The data of this first sample has been previously reported (see Mertens, Gerritsen, et al., 2020). We used the data of this first sample as a reference point for the main sample of participants. Particularly, the data of 173 participants of this initial sample could be matched to the main sample based on gender, age, education level, and region of origin. We believe that the results of this matched sample provide an interesting reference point of fear at the very beginning of the pandemic (i.e., only 2-6 days after the classification of the SARS-CoV-2 virus as a pandemic by the World Health Organization).

The main sample consisted of 2000 participants recruited through Prolific (www.prolific.co). Table S6 present the results of a small power analysis showing that this sample is sufficiently powered to detect small to moderate interaction effects between for instance region and time (corresponding to small to moderate differences between regions in their change in fear of the coronavirus across time). This sample first completed the survey in April 2020 and was then re-invited each month to complete the survey again between April and August of 2020 (with data collection taking place between the $14^{\text {th }}$ and $17^{\text {th }}$ of each month). Participants were asked again in June 2021 to fill out the questionnaires. Of the initial sample, 1050 participants $(52.5 \%)$ completed all time points during the first wave in 2020 , and 668 participants (33.4\%) completed all time points including the last one in June 2021. Table 1 provides a detailed overview of the demographics of our sample. Those who completed all time points were more likely to be older, female, and on average have a slightly higher score on fear 
of COVID-19 compared to dropouts (see Table S1 in the Supplementary Materials).

Participation in both samples was on a voluntary basis and all participants provided informed

consent. The sample size was based on practical considerations (i.e., available budget) and

maintaining a large enough sample for stable observations after attrition (Schönbrodt \& Perugini,

2013). The study was approved by the Ethics Committee of the Tilburg School of Social and

Behavioral Sciences (reference code: RP216).

Table 1. Demographic information of the respondents on baseline (April 2020; total $N=2000)$.

\begin{tabular}{|c|c|c|}
\hline & $N$ & $\%$ \\
\hline \multicolumn{3}{|l|}{ Age in years } \\
\hline $16-20$ & 315 & $15.8 \%$ \\
\hline $21-30$ & 887 & $44.4 \%$ \\
\hline $31-40$ & 478 & $23.9 \%$ \\
\hline $41-50$ & 184 & $9.2 \%$ \\
\hline $51-60$ & 90 & $4.5 \%$ \\
\hline $61-70$ & 42 & $2.1 \%$ \\
\hline $71-80$ & 4 & $0.2 \%$ \\
\hline \multicolumn{3}{|l|}{ Gender } \\
\hline Male & 1018 & $50.9 \%$ \\
\hline Female & 972 & $48.6 \%$ \\
\hline Prefer not to say & 10 & $0.5 \%$ \\
\hline \multicolumn{3}{|l|}{ Highest education } \\
\hline Less than High School & 47 & $2.4 \%$ \\
\hline High School diploma & 796 & $39.8 \%$ \\
\hline College degree & 802 & $40.1 \%$ \\
\hline Master's degree & 320 & $16.0 \%$ \\
\hline Doctorate (PhD or equivalent) & 35 & $1.8 \%$ \\
\hline \multicolumn{3}{|l|}{ Country of residence by region ${ }^{1}$} \\
\hline Asia (incl. India) & 5 & $0.3 \%$ \\
\hline Australia/New-Zealand & 37 & $1.9 \%$ \\
\hline Europe (incl. Russia) & 1419 & $71.0 \%$ \\
\hline Middle-East (incl. Israel) & 11 & $0.5 \%$ \\
\hline North-America & 497 & $24.9 \%$ \\
\hline South-America & 15 & $0.8 \%$ \\
\hline Sub-Sahara Africa & 16 & $0.8 \%$ \\
\hline \multicolumn{3}{|l|}{ Infected by the coronavirus? } \\
\hline Yes & 17 & $0.9 \%$ \\
\hline No & 1871 & $93.6 \%$ \\
\hline Unsure & 112 & $5.6 \%$ \\
\hline
\end{tabular}

Note: ${ }^{1}$ Full list of countries of residence: Australia, Austria, Belgium, Canada, Chile, Czech Republic, Denmark, Estonia, Finland, France, Germany, Greece, Hungary, Iceland, Ireland, Israel, Italy, Japan, Latvia, Mexico, Netherlands, New-Zealand, Norway, Poland, Portugal, Slovenia, South-Africa, South-Korea, Spain, Sweden, Switzerland, Turkey, United Kingdom, USA. 


\subsection{Materials \& Procedure}

\subsubsection{Measures ${ }^{1}$}

2.2.1.1. Fear of the Coronavirus Questionnaire (FCQ) (Mertens, Gerritsen, et al., 2020)

The FCQ consists of eight items (for a detailed description and overview of the items of this scale, see Mertens, Gerritsen, et al., 2020). Respondents were asked to rate their level of agreement with each statement on a 5-point Likert scale (1 = "Strongly disagree", $5=$ "Strongly agree"; possible total score range: 8-40). Examples of the items are: "I am very worried about the coronavirus", "I am taking precautions to prevent infection (e.g., washing hands, avoiding contact with people, avoiding door handles)", and "I am constantly following all news updates regarding the virus". These items were chosen because they correspond with different fear components, such as subjective experiences (worrying), attentional biases, and avoidance behaviors (Lang, 1968). The internal consistency of the FCQ was acceptable (Cronbach's alpha $=0.75$; April data). Supplementary Table S2 shows the model fit of a one-factor confirmatory factor analysis for ordered categorical items, fitted separately to the full sample and to European and North American respondents. Supplementary Tables S3 and S4 show the results of a measurement invariance analysis of the FCQ across region.

\subsubsection{Intolerance of uncertainty scale (IUS)}

\footnotetext{
${ }^{1}$ In addition to the reported questionnaires, we also collected the Penn State Worry Questionnaire (Meyer et al., 1990) and asked whether participants got vaccinated or intended to do so (the latter question was only included in the June 2021 time point). However, because the PSWQ questionnaire was not an important predictor in a previous study (Mertens et al., 2020), we did not include it in our analyses. Additionally, the analyses regarding vaccination status are not directly related to the hypotheses of the current paper and will therefore be reported in a separate paper for reasons of parsimony and consistency.
} 
Intolerance of uncertainty (IU) was measured using the IUS-12 developed and validated by Carleton et al. (2007), which assesses an individual's propensity to find uncertain situations unpleasant. It consists of 12 statements scored on 5-point Likert scales ( $1=$ "Not at all characteristic of me", 5 = "Entirely characteristic of me"). Examples of the statements are: “Unforeseen events upset me greatly", "It frustrates me not having all the information I need", and "Uncertainty keeps me from living a full life". In the current study IUS was operationalized as an individual's total score on the 12 statements. The internal consistency of this scale was excellent in the current sample (Cronbach's alpha =0.89; April data).

\subsubsection{Short Health Anxiety Inventory (SHAI)}

The SHAI was used to evaluate individuals' tendency to worry about their health (Abramowitz et al., 2007; Salkovskis et al., 2002). It consists of 18 four-choice questions. An example item is: "1 = I do not worry about my health; 2 = I occasionally worry about my health; 3 = I spend much of my time worrying about my health; 4 = I spend most of my time worrying about my health". Typically, this scale is divided into two subscales: Illness likelihood and illness severity (Alberts et al., 2013). The total scores of both subscales were used in further analyses. The internal consistency was good for the illness likelihood subscale (Cronbach's alpha $=0.89$ ) and acceptable for the illness severity subscale (Cronbach's alpha $=0.72$ ).

\subsubsection{Media exposure}

To measure voluntary exposure to news about the coronavirus, respondents were asked to answer the following question: "Have you looked up any extra information regarding the coronavirus outbreak? (not taking into account coincidentally seeing/reading about it in the news)" with yes 
or no. Furthermore, if they had looked up any information, they were asked to indicate what sources they consulted (options: "Regular newspapers/websites/TV news", "Social media (Facebook, Twitter, Instagram, ...)", "Professional websites (health institute, blogs posted by virologists/biologists, ...)", "Friends/family/acquaintances", "Online searches (e.g., through Google, Bing, Ecosia, etc.)”, “Other (please specify)”; multiple answers were possible. Dummy variables were created for each of the media sources used.

\subsubsection{General health, risk control, and risk for loved ones}

Respondents were asked to rate their general health, perceived control, and perceived risk for their loved ones using 5-point rating scales. Particularly, they were asked to answer the following question: "Overall, I would rate my general health as:" (options: "Extremely good”, "Somewhat good", "Neither good nor bad", "Somewhat bad", "Extremely bad"). Perceived control was assessed with the following question: "Overall, I believe that I can control or avoid becoming infected by the coronavirus (e.g., by limiting social contact, washing hands, wearing a face mask, etc.):" (options: "Strongly agree', "Somewhat agree", "Neither agree nor disagree", "Somewhat disagree", "Strongly disagree"). Finally, risk perception for loved ones was assessed with the following question: “Overall, I believe that people that I care about (e.g., grandparents) are at risk of becoming infected and seriously ill due to the coronavirus outbreak:" (options: "Strongly agree', "Somewhat agree", "Neither agree nor disagree", "Somewhat disagree", "Strongly disagree").

\subsubsection{Demographic information}


Respondents were asked to indicate the gender they identify with the most ("male", "female", "prefer not to say"), their age (in decade categories), their highest educational level obtained (from "less than high school degree" to "Doctorate (PhD or equivalent)"), whether they work in healthcare ("yes", "no", "unsure (please clarify)"), whether they already got infected by the virus ("yes", "no", "unsure"), and their country of residence.

\subsubsection{Survey administration}

All questionnaires were delivered in English through an online survey using the Qualtrics platform (https://www.qualtrics.com/). The survey could be completed using a personal computer/laptop, tablets, or smartphone, in approximately 15 minutes.

\subsection{Data analysis strategy}

As a reference point, we have included the matched data of the March sample in all graphs and tables. However, this data was not included in the statistical models given that we were only able to match 173 participants from this time point to the main sample, implying a drastic loss of statistical power. Nonetheless, we believe it is interesting to represent the data of this time point in the figures below given that it was collected prior to the first peak of infections in the EU and the US in April 2020. Therefore, it provides an indication of the pre-pandemic levels of fear.

Linear mixed models (LMM) were fitted to examine the level and change of fear of the coronavirus across the five monthly measurements and at one-year follow-up. Parameters were estimated using maximum likelihood estimation, and an unstructured residual covariance matrix was used to model the dependency in the repeated measurements within participants. The full 
information maximum likelihood estimator allowed including participants with one or more missing repeated observations.

The LMM predictors were entered sequentially. In model 1, the effect of time (April August 2020 + June 2021) was modeled to assess whether fear of coronavirus changed significantly over time. From model 2 onwards, three separate analyses were performed, with either region (North America or Europe, fixed predictor), personality traits (health anxiety likelihood, health anxiety severity, and intolerance of uncertainty, time-varying predictor) or media use (yes/no, fixed predictor) as the independent variables. The personality trait effects were decomposed in between-subject (person-means scores over the six measurements) and within-subjects effects (the deviation from this person-mean at each time point). In Model 3, the interaction between the predictor(s) with time was included. Lastly, in model 4 all effects were adjusted for baseline demographics (e.g., gender, age). Statistical analyses were performed using SPSS 24. $P$-values smaller than .05 were considered statistically significant. 


\subsection{Data availability.}

\section{Results}

The data files and data analysis syntax of the results reported here can be obtained through the Open Science Framework (https://osf.io/t5uvn/).

\subsection{Fear over time}

Paired-samples $t$-tests were performed to calculate the difference in fear of coronavirus scores between April and the other time measurements. These indicated that the level of fear was higher in April $2020(M=30.1, S D=5.1)$ than for May $2020(M=28.7, S D=5.6 ; t(1650)=14.8, p$ $<.001, d=0.25)$, June $2020(M=27.4, S D=6.1 ; t(1559)=22.1, p<.001, d=0.46)$, July $(M=$ $27.7, S D=6.4 ; t(1413)=18.1, p<.001, d=0.39)$, August $2020(M=27.5, S D=6.1 ; t(1287)=$ $18.8, p<.001, d=0.43)$, and June $2021(M=25.6, S D=6.4 ; t(910)=25.1, p<.001, d=0.83)$.

Additionally, the linear mixed modelling analysis showed a significant decline in fear of the coronavirus over time $(F=633.59, p<.001$; see first table line, Table 2$)$. The sharpest decline was observed from April to June 2020, after which the levels of fear stabilized and further decreased again in June 2021 (see Figure 1). 


\section{Fear of Coronavirus}

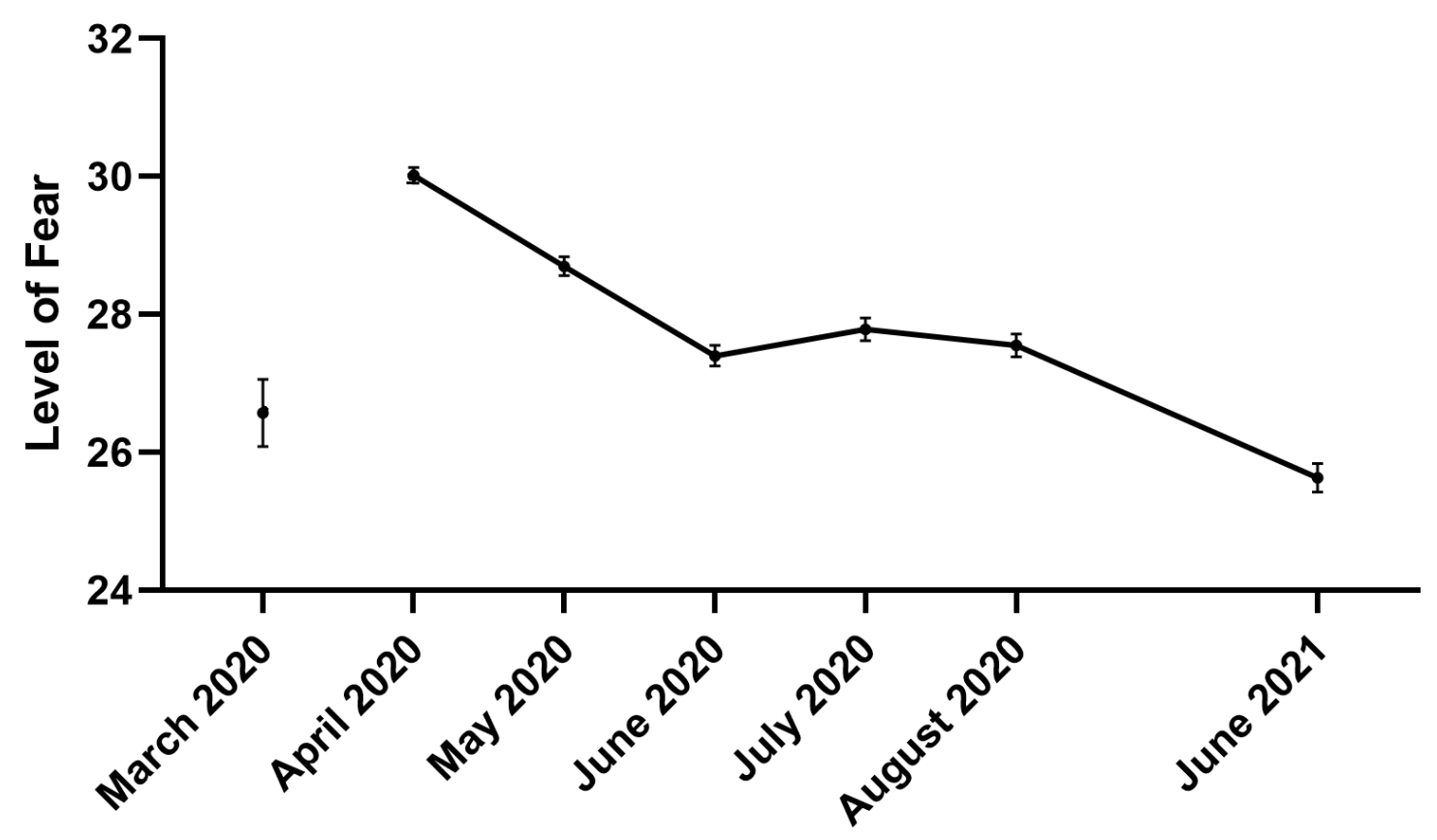

Figure 1. Change in Fear of the Coronavirus Questionnaire (FCQ) scores over time. Error bars reflected the standard error of the mean. Note: the data from March were not included in the analyses (i.e., because they were part of an independent, but matched, sample) and are therefore plotted separately in the figure.

\subsection{Effects of region}

For this analysis, we have focused on Europe and North America because we only had enough data points for these two regions ( $n=1419$ and 497, respectively), and because of the stark differences in the progression of the number of infections in these two regions during the data collection period (Nisen, 2020; Winkleman \& Santamaria, 2020). Results showed a main effect of region (Europe vs. North America) on fear of coronavirus over time in the unadjusted analyses $(F=91.91, p<.001)$. In Step 2, we added the interaction term between time and region, to account for individual differences in fear over time between regions. The interaction between time and region was significant $(F=12.35, p<.001)$, indicating a steeper decline in fear in 
North America compared to Europe (see Figure 2a). These results remained significant after adjusting for demographic variables (see Table 2). Being female, having a worse general health, having a chronic illness, and worrying about the risk of loved ones, were significantly associated with fear of the coronavirus across time. When adjusting for all confounding variables (all predictors and covariates), the main effect of region remained significant $(F=89.67, p<.001)^{2}$.

${ }^{2}$ Based on the measurement invariance analysis of the FCQ (see Section 2.2.1.1 and Tables S2-4), we found that FCQ Item 6 ("The health authorities are not doing enough to deal with the virus") was not invariant across region (Europe vs. North America). Therefore, we conducted a sensitivity analysis for the analysis involving the effects of region by repeating this analysis using the total FCQ score, excluding Item 6. Both the main effect of region $(p<.001)$ and the interaction between time and region $(p=.020)$ remained significant in the model adjusting for confounders (see Table S5 in the Supplementary Materials). 
Table 2. Results from multivariable linear mixed models for country region predicting fear of COVID-19.

\begin{tabular}{|c|c|c|c|c|c|c|c|c|c|c|c|c|}
\hline \multirow{2}{*}{$\begin{array}{l}\text { Predictor } \\
\text { Europe vs. North America }\end{array}$} & \multicolumn{3}{|c|}{ Model 1 (Time) } & \multicolumn{3}{|c|}{ Model 2 (+ Region) } & \multicolumn{3}{|c|}{ Model 3 (+ Interactions) } & \multicolumn{3}{|c|}{ Model 4 (Fully adjusted) } \\
\hline & Est. & $95 \% \mathrm{CI}$ & $\begin{array}{c}p- \\
\text { value }\end{array}$ & Est. & $95 \% \mathrm{CI}$ & $\begin{array}{c}p- \\
\text { value }\end{array}$ & Est. & $95 \% \mathrm{CI}$ & $p$-value & Est. & $95 \% \mathrm{CI}$ & $\begin{array}{c}p- \\
\text { value }\end{array}$ \\
\hline \multicolumn{13}{|l|}{ Main effects } \\
\hline Time (April 2020 - June 2021) & -.27 & {$[-0.29,-0.25]$} & $<.001$ & -.27 & {$[-0.30,-0.25]$} & $<.001$ & -.34 & {$[-0.39,-0.30]$} & $<.001$ & -.35 & {$[-0.40,-0.30]$} & $<.001$ \\
\hline Country Region (Europe) & - & - & - & -2.32 & {$[-2.80,-1.85]$} & $<.001$ & -2.59 & {$[-3.09,-2.09]$} & $<.001$ & -1.59 & {$[-3.01,-1.98]$} & $<.001$ \\
\hline \multicolumn{13}{|l|}{ Interactions } \\
\hline Time * Country Region (Europe) & - & - & - & - & - & - & .10 & {$[0.04,0.15]$} & $<.001$ & .10 & {$[0.05,0.16]$} & $<.001$ \\
\hline \multicolumn{13}{|l|}{ Demographics } \\
\hline Female gender & - & - & - & - & - & - & - & - & - & 1.12 & {$[0.69,1.55]$} & $<.001$ \\
\hline General health & - & - & - & - & - & - & - & - & - & -.62 & {$[-0.90,-0.35]$} & $<.001$ \\
\hline Chronic illness* & - & - & - & - & - & - & - & - & - & .70 & {$[0.10,1.31]$} & .023 \\
\hline Risk control & - & - & - & - & - & - & - & - & - & -.05 & {$[-0.30,0.20]$} & .706 \\
\hline Risk loved ones & - & - & - & - & - & - & - & - & - & 1.34 & {$[1.11,1.58]$} & $<.001$ \\
\hline
\end{tabular}

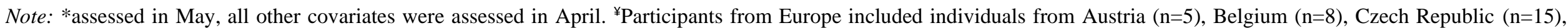

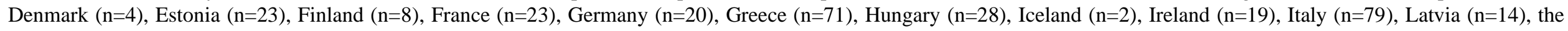

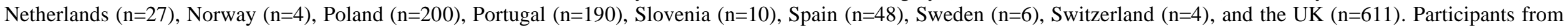
North America included individuals from Canada $(\mathrm{n}=64)$, Mexico $(\mathrm{n}=43)$, and United States of America $(\mathrm{n}=390)$. Bold values are indicative of $p<.05$. 


\subsection{Effects of anxious personality}

Results showed a main effect of between-subject differences in intolerance of uncertainty on fear of coronavirus $(F=51.43, p<.001)$, in that participants with higher intolerance of uncertainty tended to show more fear. The within-subject differences in intolerance of uncertainty scores was also significant and positive $(F=197.22, p<.001)$, indicating that an increase in intolerance of uncertainty over time was associated with more fear of coronavirus. These main effects of between- and within-subject differences were similar for illness likelihood scores $(F=73.55, p$ $<.001 ; F=45.56, p<.001)$. For illness severity, the between-subject differences on fear of coronavirus were significant and negative $(F=7.91, p=.005)$, indicating that higher illness severity scores were associated with lower fear of the coronavirus. However, the within-subject differences were non-significant $(F=.87, p=.351)$.

In Step 2, the results showed a significant and positive time*between-subject differences for illness likelihood scores interaction $(F=6.86, p=.009)$, suggesting that the decrease in fear of the coronavirus over time was less for those with higher illness likelihood scores. The results also showed a significant and negative time*within-subject differences for illness likelihood scores interaction $(F=5.55, p=.019)$, and illness severity scores interaction $(F=6.97, p$ $=.008)$. This indicates that the decrease in fear of the coronavirus over time was more pronounced for those participants whose illness likelihood and severity scores declined over time. Note however that these interaction effects were not very pronounced. No significant interactions between time and the other personality traits (between- and within-subject differences) were observed (Table 3).

The above-mentioned pattern of results was stable, even when adjusting for demographic covariates. In this model, female gender, having a chronic illness, and worrying about the risk of 
loved ones were associated with more fear of coronavirus over time (Table 3). When adjusting for all the predictors, the main effects remained similar as well. 
Table 3. Results from multivariable linear mixed models for personality traits predicting fear of COVID-19.

\begin{tabular}{|c|c|c|c|c|c|c|c|c|c|c|c|c|}
\hline \multirow{2}{*}{$\begin{array}{l}\text { Predictor } \\
\text { Anxious personality }\end{array}$} & \multicolumn{3}{|c|}{ Model 1 (Time) } & \multicolumn{3}{|c|}{ Model 2 (+ Personality) } & \multicolumn{3}{|c|}{ Model 3 (+ Interactions) } & \multicolumn{3}{|c|}{ Model 4 (Fully adjusted) } \\
\hline & Est. & $95 \% \mathrm{CI}$ & p-value & Est. & $95 \% \mathrm{CI}$ & $\begin{array}{c}\mathrm{p}- \\
\text { value }\end{array}$ & Est. & $95 \% \mathrm{CI}$ & $\begin{array}{c}\mathrm{p}- \\
\text { value }\end{array}$ & Est. & $95 \% \mathrm{CI}$ & $\begin{array}{c}\mathrm{p}- \\
\text { value }\end{array}$ \\
\hline \multicolumn{13}{|l|}{ Main effects } \\
\hline Time (April 2020 - June 2021) & -.27 & {$[-0.29,-0.25]$} & $<.001$ & -.25 & {$[-0.28,-0.23]$} & $<.001$ & -.28 & {$[-0.83,-0.37]$} & $<.001$ & -.28 & {$[-0.38,-0.18]$} & $<.001$ \\
\hline Intolerance of uncertainty between & - & - & - & .11 & {$[0.08,0.14]$} & $<.001$ & .11 & {$[0.09,0.16]$} & $<.001$ & .10 & {$[0.07,0.13]$} & $<.001$ \\
\hline Intolerance of uncertainty within & - & - & - & .11 & {$[0.10,0.13]$} & $<.001$ & .11 & {$[0.09,0.17]$} & $<.001$ & .11 & {$[0.08,0.13]$} & $<.001$ \\
\hline SHAI Illness likelihood between & - & - & - & .19 & {$[0.15,0.23]$} & $<.001$ & .17 & {$[0.08,0.18]$} & $<.001$ & .14 & {$[0.09,0.19]$} & $<.001$ \\
\hline SHAI Illness likelihood within & - & - & - & .08 & {$[0.06,0.11]$} & $<.001$ & .13 & {$[0.02,0.13]$} & .004 & .13 & {$[0.09,0.16]$} & $<.001$ \\
\hline SHAI Illness severity between & - & - & - & -.18 & {$[-0.31,-0.06]$} & .005 & -.13 & {$[-0.22,0.07]$} & .308 & -.12 & {$[-0.25,0.02]$} & .089 \\
\hline SHAI Illness severity within & - & - & - & -.03 & {$[-0.08,0.03]$} & .351 & .05 & {$[-0.17,0.08]$} & .458 & .05 & {$[-0.03,0.13]$} & .239 \\
\hline \multicolumn{13}{|l|}{ Interactions } \\
\hline Time * Intolerance of uncertainty between & - & - & - & - & - & - & $<-.01$ & {$[-0.00,0.00]$} & .445 & -.00 & {$[-0.0,0.00]$} & .368 \\
\hline Time * Intolerance of uncertainty within & - & - & - & - & - & - & $<.01$ & {$[-0.00,0.01]$} & .422 & .00 & {$[-0.00,0.01]$} & .336 \\
\hline Time * SHAI Illness likelihood between & - & - & - & - & - & - & .01 & {$[0.00,0.01]$} & .009 & .01 & {$[0.00,0.01]$} & .006 \\
\hline Time * SHAI Illness likelihood within & - & - & - & - & - & - & -.01 & {$[-0.02,-0.00]$} & .008 & -.01 & {$[-0.02,-0.00]$} & .008 \\
\hline Time * SHAI Illness severity between & - & - & - & - & - & - & -.01 & {$[-0.03,0.00]$} & .060 & -.01 & {$[-0.03,0.00]$} & .061 \\
\hline Time * SHAI Illness severity within & - & - & - & - & - & - & -.02 & {$[-0.04,-0.00]$} & .019 & -.02 & {$[-0.04,-0.00]$} & .012 \\
\hline \multicolumn{13}{|l|}{ Demographics } \\
\hline Female gender & - & - & - & - & - & - & - & - & - & .63 & {$[0.20,1.06]$} & .004 \\
\hline General health & - & - & - & - & - & - & - & - & - & -.03 & {$[-.32,0.26]$} & .860 \\
\hline Chronic illness* & - & - & - & - & - & - & - & - & - & .75 & {$[0.15,1.35]$} & .014 \\
\hline Risk control & - & - & - & - & - & - & - & - & - & .02 & {$[-0.2,0.28]$} & .862 \\
\hline Risk loved ones & - & - & - & - & - & - & - & - & - & 1.23 & {$[1.00,1.46]$} & $<.001$ \\
\hline
\end{tabular}

Note: *assessed in May, all other covariates were assessed in April. Abbreviations: SHAI = Short Health Anxiety Inventory. Bold values are indicative of $p<.05$. 


\subsection{Effects of media use}

Looking up additional information through media sources was significantly associated with increased fear of COVID-19 over time $(F=83.39, p<.001)$. In the second step, we made a distinction between the type of media (i.e., regular media, online searches, professional websites, and social media) that were consulted among those who looked up additional information. The results showed a positive main effect of consulting regular media $(F=18.55, p<.001)$, professional websites $(F=7.07, p=.008)$, and social media $(F=5.94, p=.015)$, on fear of COVID-19 across time, but not for online searches $(F=1.21, p=.272)$. This indicates that looking up information through regular media, professional websites, and social media is predictive of increased fear of COVID-19. A significant interaction with time was observed for social media use $(F=14.78, p<.001)$ in which more social media use was related to lower fear scores over time. The other media sources were unrelated to change in time (see Table 4).

When adding demographic covariates, the results remained similar. Female gender, poorer general health, having a chronic illness, and worrying about the risk of loved ones, were significant covariates of fear of coronavirus in this model (Table 4). Adjusting for the other predictors (region and anxious personality), we observed similar main effects. 
Table 4. Results from multivariable linear mixed models for media use predicting fear of COVID-19.

\begin{tabular}{|c|c|c|c|c|c|c|c|c|c|c|c|c|}
\hline \multirow{2}{*}{$\begin{array}{l}\text { Predictor } \\
\text { Media use }\end{array}$} & \multicolumn{3}{|c|}{ Model 1 (Time) } & \multicolumn{3}{|c|}{ Model 2 (+ Media use) } & \multicolumn{3}{|c|}{ Model 3 (+ Interactions) } & \multicolumn{3}{|c|}{ Model 4 (Fully adjusted) } \\
\hline & Est. & $95 \% \mathrm{CI}$ & p-value & Est. & $95 \% \mathrm{CI}$ & $\begin{array}{c}p- \\
\text { value }\end{array}$ & Est. & $95 \% \mathrm{CI}$ & $\begin{array}{c}p- \\
\text { value }\end{array}$ & Est. & $95 \% \mathrm{CI}$ & $\begin{array}{c}p- \\
\text { value }\end{array}$ \\
\hline \multicolumn{13}{|l|}{ Main effects } \\
\hline Time (April 2020 - June 2021) & -.27 & {$[-0.29,-0.25]$} & $<.001$ & -.30 & {$[-0.32,-0.27]$} & $<.001$ & -.38 & {$[-0.43,-0.33]$} & $<.001$ & -.38 & {$[-0.43,-0.3]$} & $<.001$ \\
\hline Media use & - & - & - & 2.27 & {$[1.79,2.76]$} & $<.001$ & - & - & - & - & - & - \\
\hline Regular media & - & - & - & 1.06 & {$[0.58,1.54]$} & $<.001$ & 1.09 & {$[0.59,1.60]$} & $<.001$ & 1.06 & {$[0.55,1.56]$} & $<.001$ \\
\hline Online Searches & - & - & - & .28 & {$[-0.22,0.78]$} & .272 & .43 & {$[0.10,0.95]$} & .110 & .42 & {$[-0.10,0.95]$} & .115 \\
\hline Professional websites & - & - & - & .68 & {$[0.18,1.17]$} & .008 & .73 & {$[0.21,1.25]$} & .006 & .80 & {$[0.27,1.33]$} & .003 \\
\hline Social Media & - & - & - & .58 & {$[0.11,1.05]$} & .015 & .86 & {$[0.37,1.35]$} & $<.001$ & .94 & {$[0.45,1.43]$} & $<.001$ \\
\hline \multicolumn{13}{|l|}{ Interactions } \\
\hline Regular media*Time & - & - & - & - & - & - & -.01 & {$[-0.07,0.04]$} & .633 & -.01 & {$[-0.07,0.04]$} & .698 \\
\hline Online Searches*Time & - & - & - & - & - & - & -.05 & {$[-0.11,0.00]$} & .068 & -.05 & {$[-0.11,0.00]$} & .054 \\
\hline Professional websites*Time & - & - & - & - & - & - & -.02 & {$[-0.08,0.04]$} & .517 & -.02 & {$[-0.08,0.04]$} & .516 \\
\hline Social Media*Time & - & - & - & - & - & - & -.10 & {$[-0.16,-0.05]$} & $<.001$ & -.10 & {$[-0.16,-0.05]$} & $<.001$ \\
\hline \multicolumn{13}{|l|}{ Demographics } \\
\hline Female gender & - & - & - & - & - & - & - & - & - & .97 & {$[0.50,1.43]$} & $<.001$ \\
\hline General health & - & - & - & - & - & - & - & - & - & -.60 & {$[-0.90,-0.30]$} & $<.001$ \\
\hline Chronic illness* & - & - & - & - & - & - & - & - & - & .64 & {$[0.00,1.28]$} & .050 \\
\hline Risk control & - & - & - & - & - & - & - & - & - & -.06 & {$[-0.34,0.21]$} & .659 \\
\hline Risk loved ones & - & - & - & - & - & - & - & - & - & 1.21 & {$[0.95,1.47]$} & $<.001$ \\
\hline
\end{tabular}

Note: *assessed in May, all other covariates were assessed in April. Bold values are indicative of $p<.05$. 

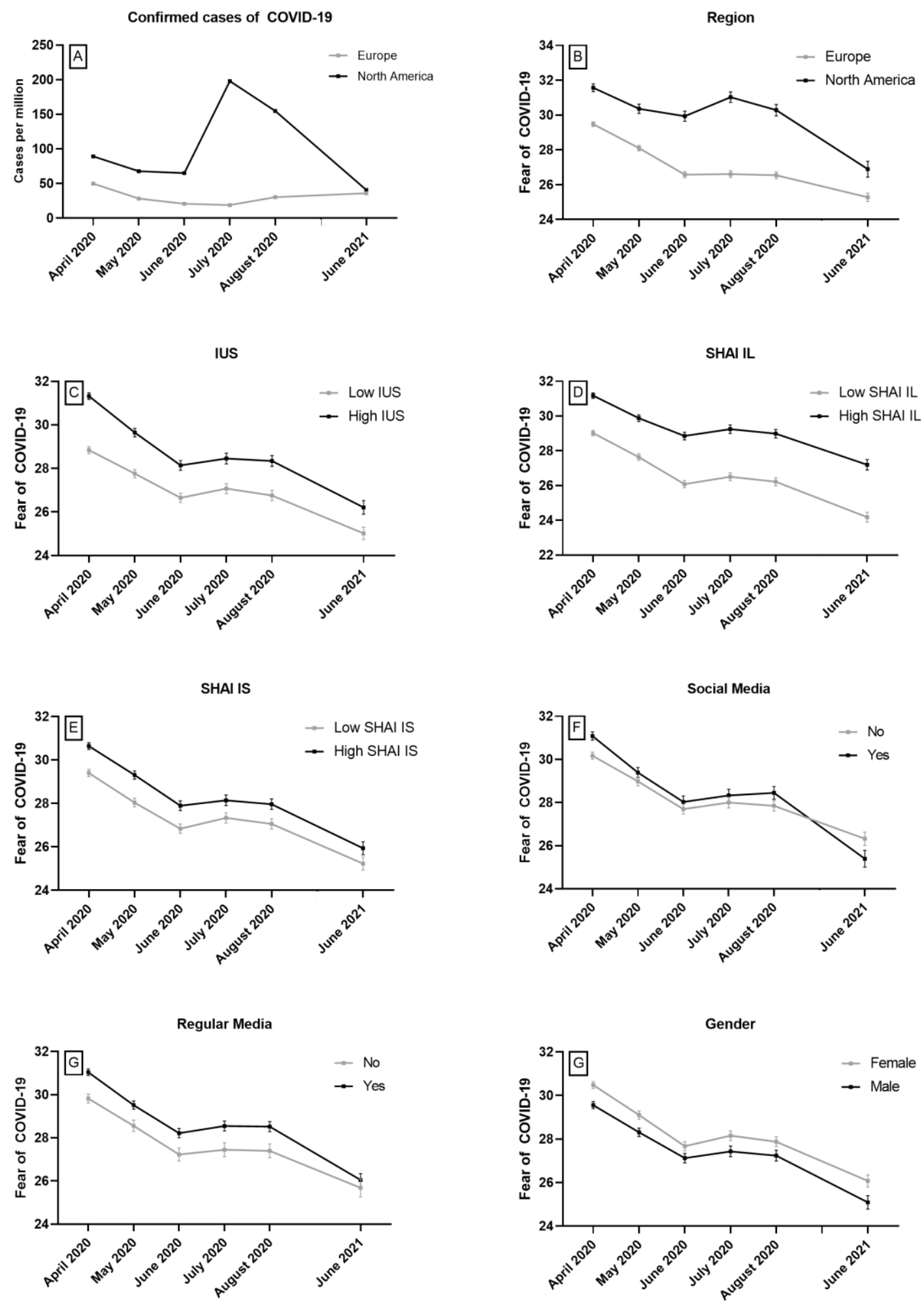

Figure 2. The number of confirmed cases per million by region (based on https://ourworldindata.org/coronavirus; A) and Fear of the Coronavirus Questionnaire scores over time (April through August 2020 + June 2021) according to different predictors $(B-H)$. Note: IUS = Intolerance of Uncertainty Scale; SHAI IL = Short Health Anxiety Inventory Illness Likelihood; SHAI IS = Short Health Anxiety Inventory Illness Severity. 


\section{Discussion}

In this study we examined the temporal changes in fear of COVID-19. We find that fear was highest in April 2020, and then gradually declined up until our last measurement point (June 2021). Furthermore, we find different predictors of elevated fear of COVID-19: Region of residence (North America), anxious personality traits, and media use. Additionally, we find that the gender, general health, and risks for loved ones are also important predictors of increased fear. Given the prior observed associations between fear of COVID-19 and stress, anxiety, and depression (Fitzpatrick et al., 2020; Lee et al., 2020; Montano \& Acebes, 2020), these factors predictive of fear of COVID-19 probably also contribute to elevated levels of psychological distress. We discuss the contribution of each of these predictors for increased fear of COVID-19 in more detail below.

First, one of the clearest findings in this study is that fear was highest around April 2020, and then gradually declined. This matches the first peak in COVID-19 infections, hospitalizations and deaths in Europe (which represented $71 \%$ of the countries in our sample) and implies that subjective fear of COVID-19 tracks the objective intensity of the pandemic quite well. Interestingly, North America had a different trajectory of the pandemic than Europe (Nisen, 2020; Winkleman \& Santamaria, 2020). The number of cases in most European countries reached their peak around April 2020 and then remained low in the summer of 2020, whereas the number of cases remained high throughout this period in North America (see Figure 2A). This is mirrored by significant differences in fear observed between Europe and North America. This suggests that people generally are quite aware of the severity of the pandemic and that fear is affected by the local intensity of the pandemic. Interestingly, the difference between the two regions became less pronounced in June 2021. Nonetheless, reported fear of COVID-19 was still 
significantly higher in North America than in Europe at this time point, even though the number of cases per million was near identical in the two regions (see Figure 2A). That fear of COVID19 remained more highly pronounced in North America compared to Europe may reflect the greater intensity of the COVID-19 pandemic throughout 2020 and/or the greater political polarization of the pandemic in North America, particularly in the US (Bruine de Bruin et al., 2020; Kerr et al., 2021).

The second major finding of our study is that anxious personality traits and female gender are predictive of increased fear of COVID-19. This fits well with previous research demonstrating that anxious personality traits and female gender are related to an increased prevalence of anxiety disorders (Carleton, 2016; McLean et al., 2011). Hence, it seems likely that the same factors associated with increased vulnerability to experiencing anxiety and fear generally are also predictive of increased levels of COVID-19 related fear. Indeed, other studies have also found that these factors are related to increased fear of COVID-19 (e.g., Broche-Pérez et al., 2020; Jungmann \& Witthöft, 2020; Satici et al., 2020; Wheaton et al., 2021). Interestingly, these differences remained present throughout the time window of this study, even though the number of cases per million were substantially declined towards the end of the study. This suggests that gender and anxious personality traits tend to predispose towards higher levels of fear and that this predisposition remains consistent, regardless of the objective risk level of the situation. Nonetheless, it is worth noting that for both women and highly anxious participants, fear of COVID-19 did substantially decrease throughout the course of the pandemic. Hence, even though they experience a generally heightened level of fear, women and individuals with higher levels of anxiety nonetheless do also experience a decline in fear levels. 
Finally, a third important finding of our study is that media usage is related to increased fear of COVID-19. This resembles the findings of previous reports for earlier pandemics (e.g., Van den Bulck \& Custers, 2009) and several recent studies (e.g., Chao et al., 2020; Garfin et al., 2020). Interestingly, though, we found a positive relationship between anxiety and both social and traditional media usage, whereas a recent study only found a relationship with social media usage (Chao et al., 2020). This difference may be because our study focused on fear specifically relating to COVID-19, whereas the Chao et al. study focused on general anxiety symptoms. Another interesting finding was that the fear-eliciting effect of media use was largely absent in June 2021, and the effect of social media use even seemed to have been reversed (see Figures $2 \mathrm{~F}$ and $2 \mathrm{G}$ ). One could stipulate that those participants who actively used social and traditional media to look up information regarding COVID-19 were better informed about the improved epidemiological situation (i.e., the decrease in number of infections and/or the effectiveness of the COVID vaccinations) and, therefore, had a more outspoken decline in their fear of COVID19 in June 2021. This provides some nuance to the common claim that media use leads to increased levels of fear and psychological distress (Lin et al., 2020; Sasaki et al., 2020). However, this explanation remains speculative as we did not measure these potential moderators (e.g., level of knowledge about the pandemic) and more systematic-experimental research on how media use influences fear and risk perceptions is needed.

Some limitations of this study are worth noting. First, we did not collect nationally representative samples for this study. Therefore, it is unclear to what extent our results can be generalized to the general populations of the countries that were included in this study. Second, we did not use scales to assess clinical levels of psychological distress (e.g., using the General 
Anxiety Disorder-7 or a similar scale; Spitzer et al., 2006). Hence, this study does not provide information about how fear of COVID-19 affected clinical levels of psychological distress. Third, the assessment of COVID-19 fear was concise. Particularly, in a previous study we found that fear of COVID-19 has four different dimensions: fear of health-related consequences, fear of supplies shortages and xenophobia, fear about socio-economic consequences, and symptoms of fear (e.g., compulsions, nightmares) (Mertens, Duijndam, et al., 2021). The FCQ only assesses fear of health-related consequences. It is unclear at this point how our results generalize to the other dimensions of COVID-19 related fear. Fourth, the temporal resolution of our study was limited. Due to budgetary constraints, we were unable to collect new data in every month throughout the time window of our study. This may have resulted in important variation in experienced fear levels not being assessed. For instance, after September 2020 several countries have had several second and even third waves in the numbers of infections, hospitalization, and deaths due to COVID-19. Most likely, this was associated with new increases in fear, yet we did not assess fear at these potentially meaningful time points. Finally, our measurement invariance analysis suggested that especially FCQ Item 6 ("The health authorities are not doing enough to deal with the virus") was not invariant across region (Europe vs. North America). Regardless of the construct fear of COVID-19, respondents in North America tended to believe that their health authorities were not doing enough to deal with the virus. Such differential item functioning may have caused slightly inflated FCQ total scores for North Americans compared to Europeans, regardless of their true score on the latent construct fear of COVID-19. Nonetheless, in a sensitivity analysis for the effects of region, we took this issue into account by repeating the analysis with the total FCQ score excluding Item 6 . We found that the pattern of results remained the same in this sensitivity analysis (see the Supplementary Materials and Footnote 2), indicating 
that the measurement invariance of Item 6 of the FCQ alone could not explain the observed regional difference (i.e., North America > Europe) in fear of COVID-19.

In conclusion, the current study investigated the levels of fear of COVID-19 in a large international study. Since April 2020, fear levels associated with this pandemic have substantially declined. We identified several relevant predictors of fear of COVID-19, such as region, anxious personality traits, gender, and media use. These results demonstrate how these different factors contribute to experiencing fear of COVID-19 and how the effects vary over time. 


\section{References}

Abramowitz, J. S., Deacon, B. J., \& Valentiner, D. P. (2007). The Short Health Anxiety Inventory: Psychometric Properties and Construct Validity in a Non-clinical Sample. Cognitive Therapy and Research, 31(6), 871-883. https://doi.org/10.1007/s10608-0069058-1

Ahorsu, D. K., Lin, C.-Y., Imani, V., Saffari, M., Griffiths, M. D., \& Pakpour, A. H. (2020). The Fear of COVID-19 Scale: Development and Initial Validation. International Journal of Mental Health and Addiction. https://doi.org/10.1007/s11469-020-00270-8

Alberts, N. M., Hadjistavropoulos, H. D., Jones, S. L., \& Sharpe, D. (2013). The Short Health Anxiety Inventory: A systematic review and meta-analysis. Journal of Anxiety Disorders, 27(1), 68-78. https://doi.org/10.1016/j.janxdis.2012.10.009

Aslam, F., Awan, T. M., Syed, J. H., Kashif, A., \& Parveen, M. (2020). Sentiments and emotions evoked by news headlines of coronavirus disease (COVID-19) outbreak. Humanities and Social Sciences Communications, 7(1), 23. https://doi.org/10.1057/s41599-020-0523-3

Asmundson, G. J. G., \& Taylor, S. (2020). How health anxiety influences responses to viral outbreaks like COVID-19: What all decision-makers, health authorities, and health care professionals need to know. Journal of Anxiety Disorders, 71, 102211. https://doi.org/10.1016/j.janxdis.2020.102211

Broche-Pérez, Y., Fernández-Fleites, Z., Jiménez-Puig, E., Fernández-Castillo, E., \& RodríguezMartin, B. C. (2020). Gender and Fear of COVID-19 in a Cuban Population Sample. International Journal of Mental Health and Addiction. https://doi.org/10.1007/s11469-02000343-8

Bruine de Bruin, W., Saw, H.-W., \& Goldman, D. P. (2020). Political polarization in US 
residents' COVID-19 risk perceptions, policy preferences, and protective behaviors. Journal of Risk and Uncertainty, 61(2), 177-194. https://doi.org/10.1007/s11166-02009336-3

Carleton, R. N. (2016). Into the unknown: A review and synthesis of contemporary models involving uncertainty. Journal of Anxiety Disorders, 39, 30-43. https://doi.org/10.1016/j.janxdis.2016.02.007

Carleton, R. N., Norton, M. A. P. J., \& Asmundson, G. J. G. (2007). Fearing the unknown: A short version of the Intolerance of Uncertainty Scale. Journal of Anxiety Disorders, 21(1), 105-117. https://doi.org/10.1016/j.janxdis.2006.03.014

Chao, M., Xue, D., Liu, T., Yang, H., \& Hall, B. J. (2020). Media use and acute psychological outcomes during COVID-19 outbreak in China. Journal of Anxiety Disorders, 74, 102248. https://doi.org/10.1016/j.janxdis.2020.102248

De Landsheer, C., \& Walburg, V. (2022). Links between rational and irrational beliefs, trait anxiety and fear of COVID 19. Psychologie Française, 67(3), 305-316. https://doi.org/10.1016/j.psfr.2022.06.005

Dong, E., Du, H., \& Gardner, L. (2020). An interactive web-based dashboard to track COVID-19 in real time. The Lancet Infectious Diseases, 20(5), 533-534. https://doi.org/10.1016/S1473-3099(20)30120-1

Fitzpatrick, K. M., Harris, C., \& Drawve, G. (2020). Fear of COVID-19 and the mental health consequences in America. Psychological Trauma: Theory, Research, Practice, and Policy, 12(S1), S17-S21. https://doi.org/10.1037/tra0000924

Garfin, D. R., Silver, R. C., \& Holman, E. A. (2020). The novel coronavirus (COVID-2019) outbreak: Amplification of public health consequences by media exposure. Health 
Psychology. https://doi.org/10.1037/hea0000875

Hamidein, Z., Hatami, J., \& Rezapour, T. (2020). How People Emotionally Respond to the News on COVID-19: An Online Survey. Basic and Clinical Neuroscience Journal. https://doi.org/10.32598/bcn.11.covid19.809.2

Harper, C. A., Satchell, L. P., Fido, D., \& Latzman, R. D. (2020). Functional Fear Predicts Public Health Compliance in the COVID-19 Pandemic. International Journal of Mental Health and Addiction, 70 3)(5), 1. https://doi.org/10.1007/s11469-020-00281-5

Jungmann, S. M., \& Witthöft, M. (2020). Health anxiety, cyberchondria, and coping in the current COVID-19 pandemic: Which factors are related to coronavirus anxiety? Journal of Anxiety Disorders, 73, 102239. https://doi.org/10.1016/j.janxdis.2020.102239

Kerr, J., Panagopoulos, C., \& van der Linden, S. (2021). Political polarization on COVID-19 pandemic response in the United States. Personality and Individual Differences, 179, 110892. https://doi.org/10.1016/j.paid.2021.110892

Lang, P. J. (1968). Fear reduction and fear behavior: Problems in treating a construct. In J. M. Schlien (Ed.), Research in psychotherapy (pp. 90-103). American Psychological Association.

Lee, S. A., Jobe, M. C., Mathis, A. A., \& Gibbons, J. A. (2020). Incremental validity of coronaphobia: Coronavirus anxiety explains depression, generalized anxiety, and death anxiety. Journal of Anxiety Disorders, 74, 102268.

https://doi.org/10.1016/j.janxdis.2020.102268

Lin, C.-Y., Broström, A., Griffiths, M. D., \& Pakpour, A. H. (2020). Investigating mediated effects of fear of COVID-19 and COVID-19 misunderstanding in the association between problematic social media use, psychological distress, and insomnia. Internet Interventions, 
21, 100345. https://doi.org/10.1016/j.invent.2020.100345

Lu, W., Wang, H., Lin, Y., \& Li, L. (2020). Psychological status of medical workforce during the COVID-19 pandemic: A cross-sectional study. Psychiatry Research, 288, 112936. https://doi.org/10.1016/j.psychres.2020.112936

McLean, C. P., Asnaani, A., Litz, B. T., \& Hofmann, S. G. (2011). Gender differences in anxiety disorders: Prevalence, course of illness, comorbidity and burden of illness. Journal of Psychiatric Research, 45(8), 1027-1035. https://doi.org/10.1016/j.jpsychires.2011.03.006

Mertens, G., Duijndam, S., Smeets, T., \& Lodder, P. (2021). The latent and item structure of COVID-19 fear: A comparison of four COVID-19 fear questionnaires using SEM and network analyses. Journal of Anxiety Disorders, 81, 102415. https://doi.org/10.1016/j.janxdis.2021.102415

Mertens, G., Gerritsen, L., Duijndam, S., Salemink, E., \& Engelhard, I. M. (2020). Fear of the coronavirus (COVID-19): Predictors in an online study conducted in March 2020. Journal of Anxiety Disorders, 102258. https://doi.org/10.1016/j.janxdis.2020.102258

Meyer, T. J., Miller, M. L., Metzger, R. L., \& Borkovec, T. D. (1990). Development and validation of the penn state worry questionnaire. Behaviour Research and Therapy, 28(6), 487-495. https://doi.org/10.1016/0005-7967(90)90135-6

Montano, R. L. T., \& Acebes, K. M. L. (2020). Covid stress predicts depression, anxiety and stress symptoms of Filipino respondents. International Journal of Research in Business and Social Science (2147- 4478), 9(4), 78-103. https://doi.org/10.20525/ijrbs.v9i4.773

Nisen, M. (2020). A Horrifying U.S. Covid Curve Has a Simple Explanation. Bloomberg Opinion. https://www.bloomberg.com/opinion/articles/2020-06-26/coronavirus-ahorrifying-rise-in-u-s-covid-cases-is-explained 
Salkovskis, P. M., Rimes, K. A., Warwick, H. M. C., \& Clark, D. M. (2002). The Health Anxiety Inventory: development and validation of scales for the measurement of health anxiety and hypochondriasis. Psychological Medicine, 32(05).

https://doi.org/10.1017/S0033291702005822

Sasaki, N., Kuroda, R., Tsuno, K., \& Kawakami, N. (2020). Exposure to media and fear and worry about COVID-19. Psychiatry and Clinical Neurosciences, 74(9), 501-502. https://doi.org/10.1111/pcn.13095

Satici, B., Saricali, M., Satici, S. A., \& Griffiths, M. D. (2020). Intolerance of Uncertainty and Mental Wellbeing: Serial Mediation by Rumination and Fear of COVID-19. International Journal of Mental Health and Addiction. https://doi.org/10.1007/s11469-020-00305-0

Schönbrodt, F. D., \& Perugini, M. (2013). At what sample size do correlations stabilize? Journal of Research in Personality, 47(5), 609-612. https://doi.org/10.1016/j.jrp.2013.05.009

Sciensano. (2020). COVID-19 gezondheidsenquête: Enkele voorlopige resultaten. https://www.sciensano.be/sites/www.wiv-isp.be/files/report_final_nl_0.pdf

Spitzer, R. L., Kroenke, K., Williams, J. B. W., \& Löwe, B. (2006). A Brief Measure for Assessing Generalized Anxiety Disorder. Archives of Internal Medicine, 166(10), 1092. https://doi.org/10.1001/archinte.166.10.1092

Taylor, S., Landry, C. A., Paluszek, M. M., Rachor, G. S., \& Asmundson, G. J. G. (2020). Worry, avoidance, and coping during the COVID-19 pandemic: A comprehensive network analysis. Journal of Anxiety Disorders, 76, 102327. https://doi.org/10.1016/j.janxdis.2020.102327

Troisi, A., Nanni, R. C., Riconi, A., Carola, V., \& Di Cave, D. (2021). Fear of COVID-19 among Healthcare Workers: The Role of Neuroticism and Fearful Attachment. Journal of Clinical 
Medicine, 10(19), 4358. https://doi.org/10.3390/jcm10194358

Van den Bulck, J., \& Custers, K. (2009). Television exposure is related to fear of avian flu, an Ecological Study across 23 member states of the European Union. The European Journal of Public Health, 19(4), 370-374. https://doi.org/10.1093/eurpub/ckp061

Wheaton, M. G., Messner, G. R., \& Marks, J. B. (2021). Intolerance of uncertainty as a factor linking obsessive-compulsive symptoms, health anxiety and concerns about the spread of the novel coronavirus (COVID-19) in the United States. Journal of Obsessive-Compulsive and Related Disorders, 28, 100605. https://doi.org/10.1016/j.jocrd.2020.100605

Winkleman, A., \& Santamaria, C. (2020). The Graphic Truth: Two different pandemics - EU vs US. GZERO. https://www.gzeromedia.com/the-graphic-truth-two-different-pandemics-euvs-us 